\title{
Retrospective study of osteoradionecrosis in the jaws of patients with head and neck cancer
}

\author{
Brena Rodrigues Manzano, Natália Garcia Santaella, Marco Aurélio Oliveira, \\ Cássia Maria Fischer Rubira, Paulo Sérgio da Silva Santos \\ Departament of Surgery, Stomatology, Pathology and Radiology, Bauru School of Dentistry, University of São Paulo, Bauru, Brazil
}

\begin{abstract}
J Korean Assoc Oral Maxillofac Surg 2019;45:21-28)
Objectives: Osteoradionecrosis (ORN) is one of the most severe complications resulting from radiotherapy (RT) in patients with head and neck cancer (HNC). It is characterized by persistent exposed and devitalized bone without proper healing for greater than 6 months after a high dose of radiation in the area. To describe the profile and dental management of ORN in HNC patients undergoing RT in an oncological clinical research center.

Materials and Methods: A retrospective descriptive study was performed to analyze dental records from HNC patients with ORN treated at an oncological clinical research center from 2013 to 2017. A total of 158 dental records for HNC patients were selected from a total of 583 records. Afterwards, this number was distributed to three examiners for manual assessments. Each examiner was responsible for selecting dental records that contained an ORN description, resulting in 20 dental records.

Results: Mean patient age was 60.3 years with males being the most affected sex $(80.0 \%)$. The most affected area was the posterior region of the mandible $(60.0 \%)$ followed by the anterior region of the mandible $(20.0 \%)$ and the posterior region of the maxilla (10.0\%). The factors most associated with ORN were dental conditions (70.0\%) followed by isolated systemic factors (10.0\%) and tumor resection (5.0\%). There was total exposed bone closure in $50.0 \%$ of cases. The predominant treatment was curettage associated with chlorhexidine $0.12 \%$ irrigation (36.0\%).

Conclusion: Poor dental conditions were related to ORN occurrence. ORN management through less invasive therapies was effective for the closure of exposed bone areas and avoidance of infection.
\end{abstract}

Key words: Osteoradionecrosis, Head and neck neoplasms, Mandible, Conservative treatment, Maxilla [paper submitted 2018. 1. 31 / revised 1st 2018. 4. 5, 2nd 2018. 6. 5 / accepted 2018. 6. 8]

\section{Introduction}

Osteoradionecrosis (ORN) is one of the most severe complications resulting from radiotherapy (RT) in patients with head and neck cancer (HNC) and can occur between four to twenty-four months after $\mathrm{RT}^{1}$. In the literature, there are several definitions for ORN that are based mainly on clinical findings when, after head and neck radiation, the bone becomes exposed and devitalized, persisting without proper

\section{Paulo Sérgio da Silva Santos}

Departament of Surgery, Stomatology, Pathology and Radiology, Bauru School of Dentistry, University of São Paulo, Al. Octávio Pinheiro Brisola, 9-75, Bauru 17012-901, São Paulo, Brazil

TEL: +55-14-3226-6113 FAX: +55-14-3235-8258

E-mail:paulosss@fob.usp.br

ORCID: https://orcid.org/0000-0002-0674-3759

(C) This is an open-access article distributed under the terms of the Creative Commons Attribution Non-Commercial License (http://creativecommons.org/ licenses/by-nc/4.0/), which permits unrestricted non-commercial use, distribution, and reproduction in any medium, provided the original work is properly cited. Copyright (C) 2019 The Korean Association of Oral and Maxillofacial Surgeons. All rights reserved. repair for a 3 to 6 month period, resulting in $\mathrm{ORN}^{2-4}$. Firstly described by Regaud ${ }^{5}$ in 1922, ORN involves a combination of four factors: radiation, hypoxia, hypovascularization, and hypocellularization resulting in bone necrosis. The mandible is the most affected bone in head and neck radiation patients $^{6-8}$.

ORN can occur spontaneously or due to local factors including tumor localization, dose and/or type of $\mathrm{RT}^{9}$, trauma due to dental prosthetics, dental surgeries before, during, or after RT, deficient oral hygiene, and local infections including periodontal disease and dentoalveolar abscesses ${ }^{9-12}$. Arteriopathies, diabetes, alcoholism, and malnutrition are systemic factors that contribute to $\mathrm{ORN}^{13}$. The diagnosis of this complication is based on clinical characteristics including bone exposure, infection, halitosis, fistula, pathological fracture, and local pain ${ }^{14}$. In asymptomatic cases, ORN can be presumptively diagnosed with radiographic exams ${ }^{15}$.

Once the cause of ORN is determined, various types of treatment can be employed, including irrigation with 
chlorhexidine $0.12 \%$, use of antibiotics, and surgical intervention ${ }^{1,16,17}$. Surgical resection and hyperbaric oxygen therapy (HOT) have been reported to be therapies of choice ${ }^{18}$, although perhaps the maximum benefit is obtained through a combination of various therapy strategies ${ }^{1,16,17}$. Currently there is no gold standard treatment for ORN nor widely accepted guidelines. Early diagnosis and oral condition monitoring are crucial for the prevention and successful treatment of ORN ${ }^{16}$.

Considering how few studies have evaluated the conduct of clinical protocols for ORN, the present study aimed to describe the profile and dental management of ORN in patients undergoing head and neck RT in an oncological clinical research center.

\section{Materials and Methods}

A retrospective descriptive study was performed using dental record data of patients with ORN treated in an oncological clinical research center. At first, a search was performed in the electronic registration system containing 583 dental records of all the treated patients. Afterwards, HNC patients were selected, totaling 158 records which were distributed to three examiners for manual assessment. Each examiner was then responsible for selecting dental records that contained a description of ORN, resulting in a final total of 20 dental records.(Fig. 1)

From the 20 dental records of patients with ORN, information was collected regarding their age, sex, type and/or HNC localization, cancer stage, type of treatment, dose, and RT type. Regarding ORN, the localization, time between the last dental appointment and first description of ORN, predisposing factors for ORN, type of treatments administered, and outcomes between the first and last description of ORN were considered. In addition, ORN cases were classified according to the Støre and Boysen ${ }^{18}$ score based on clinical characteristics described in the dental records. Following this score, isolated mucosal defects were classified as stage 0 (zero); cases with radiological evidence of necrotic bone with intact mucosa were classified as stage I; cases with exposed bone and radiological evidence were classified as stage II; and cases with exposed bone, radiological evidence, extraoral fistula, and infection were classified as stage III. All patients signed the informed consent form in accordance with the Declaration of Helsinki.

\section{Results}

In a 4 year period (2013-2017), 158 individuals with HNC were treated at the Clinical Research Center of Bauru School of Dentistry, University of São Paulo (Bauru, Brazil). One hundred thirty-nine patients $(88.0 \%)$ received RT in the head and neck region and 20 of them were diagnosed with ORN. Sixteen patients $(80.0 \%)$ with ORN were male and 4 patients $(20.0 \%)$ were female. The mean age was 60.3 years with a range from 29 to 85 years.(Table 1)

The most common types of cancer in patients who underwent head and neck RT were oropharyngeal (38.1\%) and oral (31.7\%) cancers.(Table 2) There was a higher incidence of ORN in individuals with squamous cell carcinoma (SCC) on the floor of the mouth at $70.0 \%$ followed by the alveolar ridge SCC with $50.0 \%$ of cases in patients with oral cancer. Patients with oral and oropharyngeal cancers developed ORN $50.0 \%$ of the time.(Table 3 )

Related to antineoplastic treatment modalities, of the 11 patients who underwent only RT, 6 developed ORN; of the 32 patients who underwent RT associated with chemotherapy and surgery, 6 developed ORN; of the 28 patients who underwent RT associated with surgery, 4 developed ORN; and of the 60 patients who underwent RT and chemotherapy, 4 developed ORN. The majority of ORN cases occurred in individuals who underwent conventional/cobalt RT (10 cases).

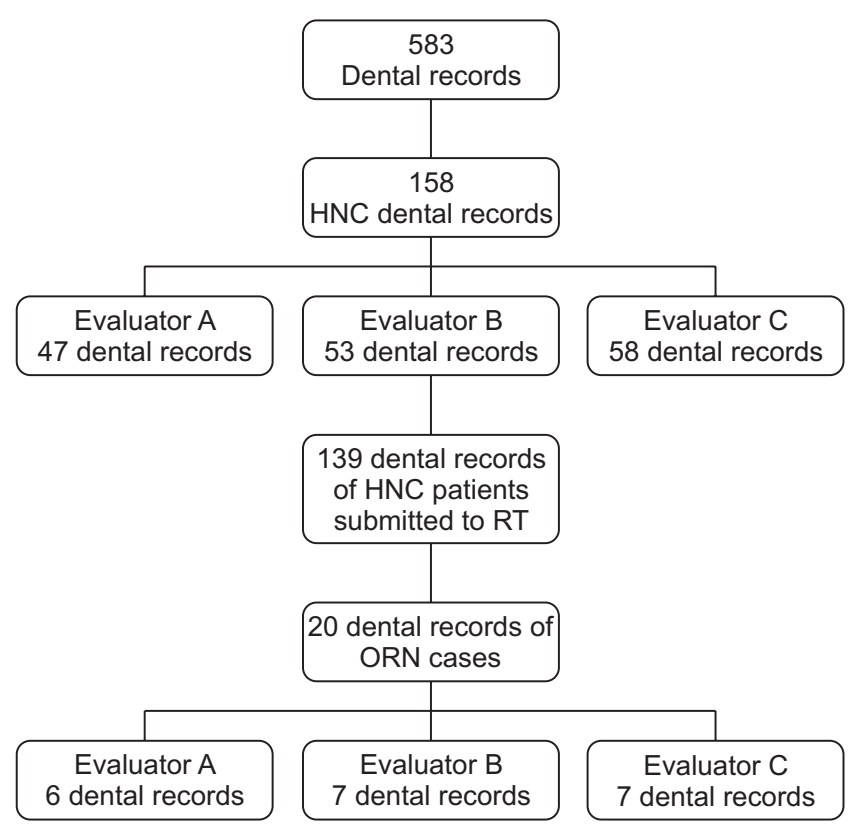

Fig. 1. Flow chart of study design. (HNC: head and neck cancer, $\mathrm{RT}$ : radiotherapy, ORN: osteoradionecrosis) Brena Rodrigues Manzano et al: Retrospective study of osteoradionecrosis in the jaws of patients with head and neck cancer. J Korean Assoc Oral Maxillofac Surg 2019 


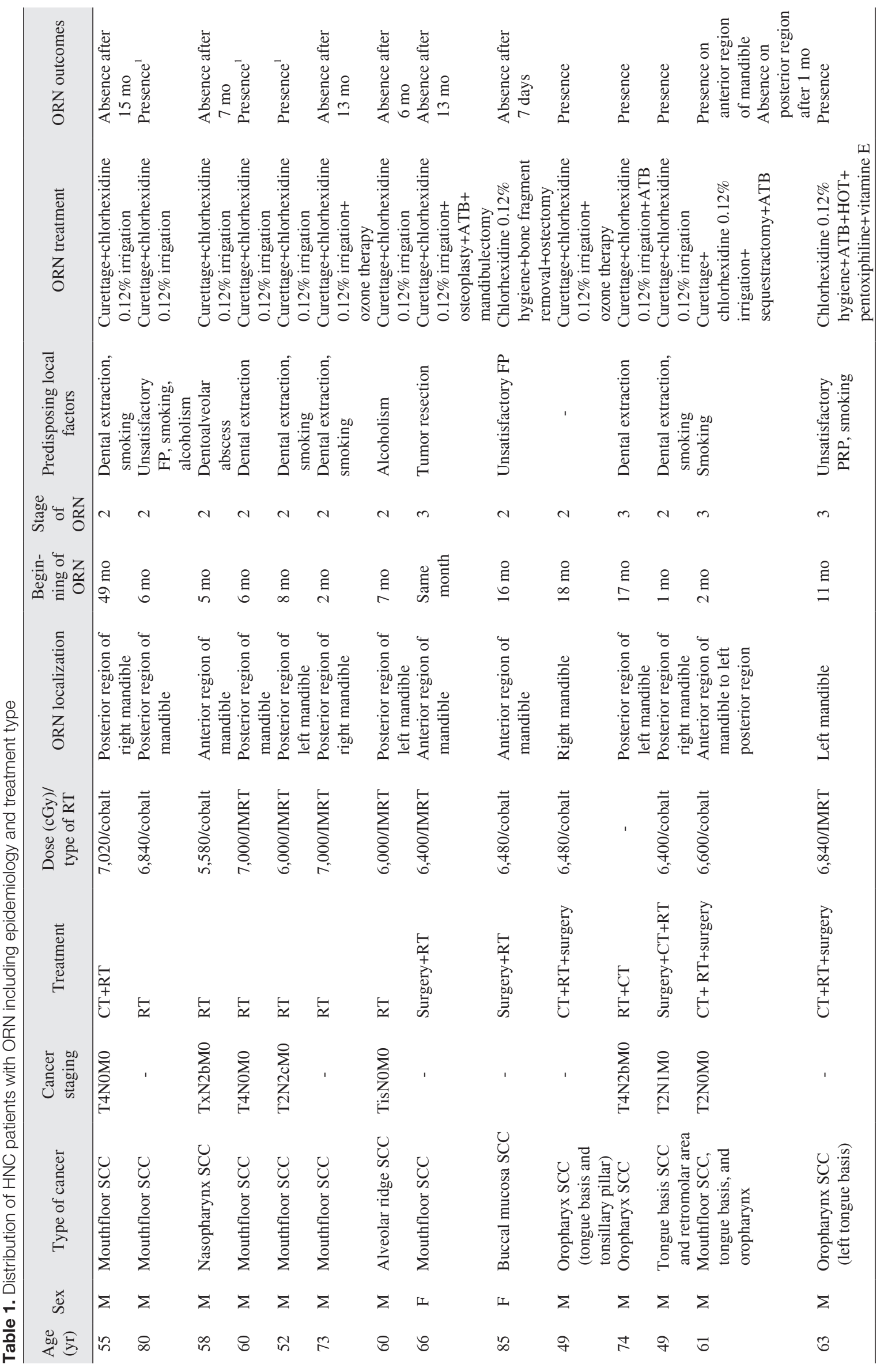




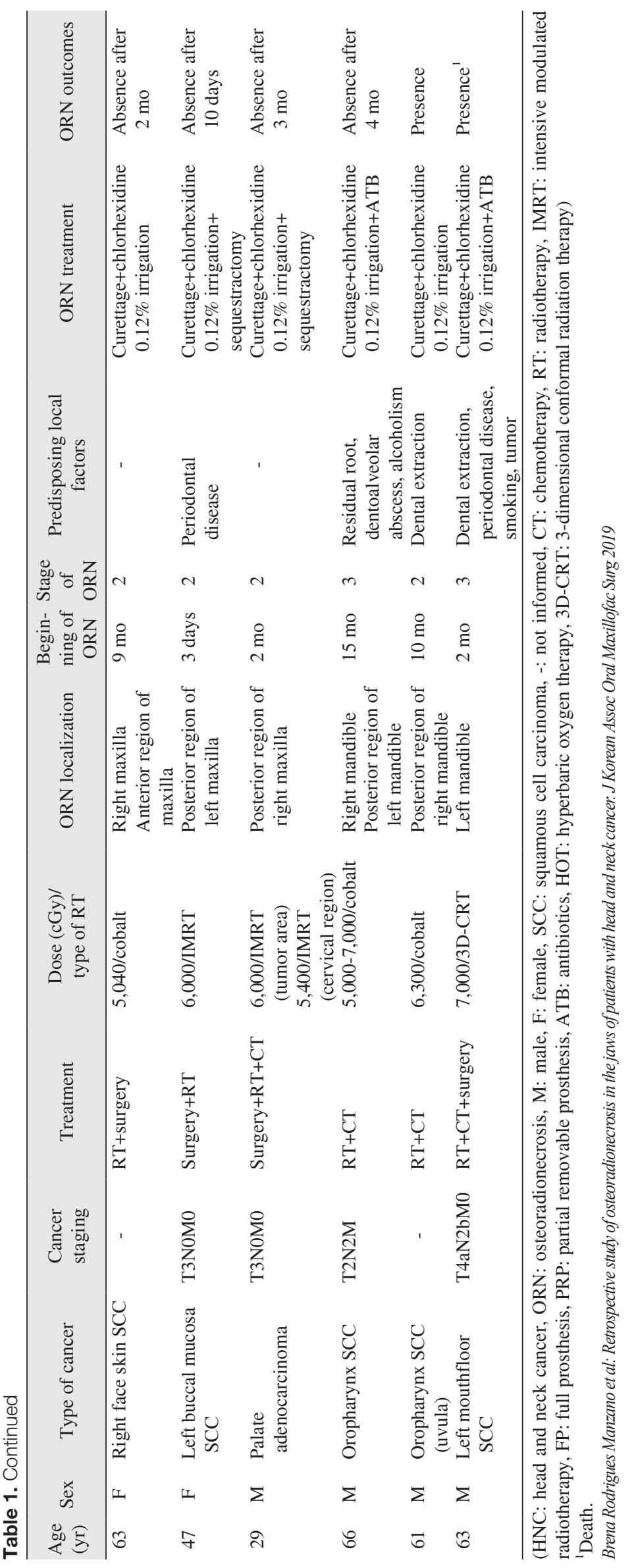


Table 2. Tumor sites in 139 patients irradiated in the head and neck area

\begin{tabular}{lc}
\hline \multicolumn{1}{c}{ Site } & Value \\
\hline Oropharynx & $53(38.1)$ \\
Oral cavity & $44(31.7)$ \\
Larynx & $20(14.4)$ \\
Hypopharynx & $4(2.9)$ \\
Nasopharynx & $8(5.8)$ \\
Others $^{1}$ & $12(8.6)$ \\
\hline
\end{tabular}

${ }^{1}$ Tumors of the parotid, maxillary sinus, skin, brain, thyroid, and hypophysis.

Values are presented as number (\%).

Brena Rodrigues Manzano et al: Retrospective study of osteoradionecrosis in the jaws of patients with head and neck cancer.J Korean Assoc Oral Maxillofac Surg 2019

Table 3. Incidence of ORN in irradiated HCN according to tumor location

\begin{tabular}{lr}
\hline \multicolumn{1}{c}{ Primary tumor } & \multicolumn{1}{c}{ Value } \\
\hline Oral cancer $(\mathrm{n}=11)$ & $7 / 10(70.0)$ \\
Mouthfloor SCC & $1 / 2(50.0)$ \\
Alveolar ridge SCC & $2 / 7(28.6)$ \\
Buccal mucosa SCC & $1 / 9(11.1)$ \\
Palate adenocarcinoma & $1 / 1(100)$ \\
Skin SCC & $2 / 4(50.0)$ \\
Oral cancer+oropharynx cancer & $1 / 8(12.5)$ \\
Nasopharynx cancer & $5 / 48(10.4)$ \\
Oropharynx & \\
\hline
\end{tabular}

(ORN: osteoradionecrosis, HCN: head and neck cancer, SCC: squamous cell carcinoma)

Values are presented as number (ORN/total tumors) (\%).

Brena Rodrigues Manzano et al: Retrospective study of osteoradionecrosis in the jaws of patients with head and neck cancer.J Korean Assoc Oral Maxillofac Surg 2019

The type of RT was not reported in 18 patients who underwent RT in the head and neck region, and only one of these cases presented with ORN.(Table 4) The dose of RT varied between 5,000 cGy and 7,020 cGy.(Table 1)

The most common ORN site was the posterior region of the mandible, with 12 individuals $(60.0 \%)$, followed by the anterior region of the mandible with 4 cases $(20.0 \%)$, posterior maxilla with 2 cases $(10.0 \%), 1$ case $(5.0 \%)$ in the anterior to posterior maxilla, and 1 case $(5.0 \%)$ that affected the anterior and posterior regions of the mandible. Regarding ORN stage, 14 out of the $20(70.0 \%)$ cases were classified as stage 2 and 6 out of the 20 cases $(30.0 \%)$ were classified as stage 3.(Table 1)

Among the ORN predisposing factors, 14 cases $(70.0 \%)$ were related to dental conditions (dental extractions, dentoalveolar abscesses, periodontal disease, dental prosthetics, and residual roots), 2 cases $(10.0 \%)$ were associated with systemic factors (tobacco and alcohol use), 1 case (5.0\%) was associated with surgery for tumor removal, and it was not possible to find descriptions of associated predisposing factors in 3 cases $(15.0 \%)$. Dental extractions and tobacco use
Table 4. Incidence of ORN according to antineoplastic treatment

\begin{tabular}{lc}
\hline \multicolumn{1}{c}{ Radiotherapy } & Value \\
\hline Modality & \\
RT & $6 / 11(54.5)$ \\
RT+CT+surgery & $6 / 32(18.8)$ \\
RT+surgery & $4 / 28(14.3)$ \\
RT+CT & $4 / 60(6.7)$ \\
Type of RT & \\
Cobalt/conventional & $10 / 54(18.5)$ \\
IMRT & $8 / 53(15.1)$ \\
3D-CRT & $1 / 14(7.1)$ \\
Not informed & $1 / 18(5.6)$ \\
\hline
\end{tabular}

(ORN: osteoradionecrosis, RT: radiotherapy, CT: chemotherapy, IMRT: intensive modulated radiotherapy, 3D-CRT: three-dimensional conformal radiation therapy)

Values are presented as number (ORN/antineoplasic treatment) (\%). Brena Rodrigues Manzano et al: Retrospective study of osteoradionecrosis in the jaws of patients with head and neck cancer. J Korean Assoc Oral Maxillofac Surg 2019

were present in 8 cases $(40.0 \%)$, followed by badly adapted prosthetics and alcohol use in 3 cases $(15.0 \%)$, and residual roots, dentoalveolar abscesses, and dehiscences following tumor removal in 1 case $(5.0 \%)$ each. ORN minimal clinical presentation time was immediate in 2 cases $(10.0 \%)$, and the larger clinical presentation time after RT totaled 49 months with an average of 6.5 months. Half of the ORN cases $(50.0 \%)$ were diagnosed 6 months after RT and after 2 months for 4 cases $(20.0 \%)$. (Table 1$)$

Many different approaches were used for ORN treatment with the most frequent ones involving curettage and $0.12 \%$ chlorhexidine irrigation in 9 cases $(45.0 \%), 2$ cases $(10.0 \%)$ of sequestrectomy associated with curettage and $0.12 \%$ chlorhexidine irrigation, and 2 cases $(10.0 \%)$ of curettage associated with $0.12 \%$ chlorhexidine irrigation and antibiotic therapy. The other associated treatment modalities (1 per case) are presented in Table 1.

ORN outcomes included 10 cases $(50.0 \%)$ that resulted in closure of the exposed bone and in 1 case $(5.0 \%)$ presenting in the anterior and posterior regions of the mandible, there was only posterior region closure. In 4 cases $(36.0 \%)$ where there was bone exposure closure/epithelialization, the treatment modality used was curettage associated with $0.12 \%$ chlorhexidine irrigation.(Table 1) The other closure cases encompassed several treatment types aforementioned.

\section{Discussion}

In the present study, ORN was found more often in elderly (61-85 years) males (80.0\%) with an average age of 60.6 years; a little older than findings from previous studies ${ }^{19,20}$ $\left(54.0^{21}, 55.2^{22}\right.$, and $57.8^{19}$ years). ORN was commonly found 
in patients with SCC on the floor of the mouth (70.0\%) in our study ${ }^{9,22}$.

Complications resulting from RT are more frequent in irradiated regions, resulting in ORN risk factors based on RT type and radiation dose. Therapy with intensive modulated radiotherapy (IMRT) presents an advantage in ORN prevention, since the ORN rate with IMRT (4\%) is lower compared to three-dimensional (3D) conformal radiation therapy (3DCRT) $(19 \%)^{22-26}$. In this study, most individuals with ORN underwent conventional RT (18.5\%) and IMRT (15.1\%) with only 1 case (7.1\%) receiving 3D-CRT.(Table 4) Doses higher than 5,000 cGy also raise the risk of ORN, with greater risk seen in doses higher than $6,000 \mathrm{cGy}^{27,28}$. ORN occurred in irradiated fields with doses ranging from 5,000 to 7,020 cGy with more frequent total doses of 6,000 cGy (4/20) and 7,000 cGy (4/20). ORN occurred independent of the treatment modality (RT and/or CT and/or surgery) and conveyed the fact that higher radiation doses contributed significantly to ORN.

When ORN occurs in the context of dental conditions associated with systemic factors (e.g., tobacco and alcohol use), including dental extractions, infections or abscesses, and trauma caused by dental prosthetics in the irradiated field, there is an increased risk for ORN ${ }^{24,29-32}$ even though ORN can occur spontaneously. In this study, ORN was found more frequently in the mandible $(80.0 \%)$, which is the region adjacent to the location of most tumors (oral floor, tongue, and retromolar trigone region), but was also present in the maxilla (10.0\%), localized next to tumors. These regions supposedly received direct radiation in high doses, seemingly the determinant factor for ORN in this study. The concept of bone density differences and blood supply between the maxilla and mandible becomes secondary.

Most ORN cases in this study were related to dental conditions $(70.0 \%)$ with only 2 cases $(10.0 \%)$ not being related to local predisposing factors, where ORN could have possibly occurred spontaneously which would be contrary to previous studies in which $79 \%{ }^{23}$ and $82 \%{ }^{33}$ of the ORN cases occurred spontaneously. Among the dental conditions, dental extractions and tobacco use were the predisposing factors more often associated $(40.0 \%)$ with ORN, followed by trauma and alcohol use (15.0\%). In another study, a low ORN rate (1.7\%) related to dental extractions was found ${ }^{23}$. However, the association with tobacco and alcohol use increased ORN risk and severity ${ }^{27,22}$. The time for ORN development after the end of RT was usually within 3 years, and varied from 0 months to 192 months in cases of chronic trauma ${ }^{9,34}$. In this study, half $(50.0 \%)$ of the ORN cases occurred 6 months after the end of
RT and ranged between 0 to 49 months with an average (6.5 years) below the one found in previous studies ( 8 months $)^{21,22}$.

Challenges for daily clinical assessments include case identification, pain source, and differentiation of ORN clinical signs when it is still in its initial stages from other side effects resulting from SCC treatment that can affect the oral mucosa, including oral mucositis ${ }^{23}$. Furthermore, it is difficult to identify radiographic alteration signs for ORN since they are only detectable when $30 \%$ to $40 \%$ of the bone density is compromised $^{18,35}$. These facts can explain the lack of description in the medical records of this study as well as signs of ORN initial stages ( 0 or 1 ), thus making it impossible to perform ORN retrospective ratings for stages other than stages 2 (70.0\%) and $3(30.0 \%)$ which had clinical sign descriptions.

ORN treatment is difficult, involves a combination of therapies, depends on available therapeutic resources, and relies on patient compliance with instructions and their individual biological response. For many researchers, conservative treatment is performed only in small ORN areas since for more advanced conditions, surgical resection is considered more efficient ${ }^{1}$. The conservative and surgical approach associated with HOT is well documented ${ }^{1,19,36}$. A less invasive option for ORN control and healing involves $0.12 \%$ chlorhexidine which, when administered topically, acts as a bactericide against gram-positive and gram-negative microorganisms and some yeasts. Despite exhibiting good results when associated with superficial necrotic bone curettage, there is still no protocol for the use of chlorhexidine for ORN treatment ${ }^{1,17}$.

Less invasive treatment options were the first choice for ORN cases in this study. Even for more advanced cases, $0.12 \%$ chlorhexidine irrigation was administered. For all stage 3 ORN cases, the use of an associated systemic antimicrobial was introduced based on the presence of local suppuration. There was epithelialization in $8(57 \%)$ stage 2 ORN cases and in $2(33 \%)$ stage 3 ORN cases. In one case, there was total ORN resolution due to surgical resection. Bone exposition closure was observed in most cases $(11 / 20)$ in this study which were treated with less invasive therapies.

\section{Conclusion}

Dental extractions, dentoalveolar abscesses, and ill-fitting dental prosthetics in directly radiated regions predisposed the areas to ORN. ORN management through less invasive therapies was effective for the treatment and control of ORN. 


\section{ORCID}

Brena Rodrigues Manzano, https://orcid.org/0000-00030412-2243

Natália Garcia Santaella, https://orcid.org/0000-0002-70208697

Marco Aurélio Oliveira, https://orcid.org/0000-0003-17533949

Cássia Maria Fischer Rubira, https://orcid.org/0000-00032119-1144

Paulo Sérgio da Silva Santos, https://orcid.org/0000-00020674-3759

\section{Authors' Contributions}

B.R.M., M.A.O., and N.G.S. participated in data collection and wrote the manuscript. P.S.S.S. participated in the study design and coordination. B.R.M. and N.G.S. performed the statistical analysis. C.M.F.R. helped to draft the manuscript. All authors read and approved the final manuscript.

\section{Acknowledgements}

We appreciate help with the preparation of the images by Marco Aurélio Rosi de Podestá and Eduardo Stedile Fiamoncini.

\section{Ethics Approval and Consent to Participate}

This study was a restrospective study with only archived records and as stated in the standards set forth in the instruction manual for submitting this journal, only experimental studies need the approval of the Institutional Review Board (IRB). Therefore, it can be justify the exemption of IRB approval for this study.

\section{Conflict of Interest}

No potential conflict of interest relevant to this article was reported.

\section{References}

1. Nadella KR, Kodali RM, Guttikonda LK, Jonnalagadda A. Osteoradionecrosis of the jaws: clinico-therapeutic management: a literature review and update. J Maxillofac Oral Surg 2015;14:891-901.

2. Wong JK, Wood RE, McLean M. Conservative management of osteoradionecrosis. Oral Surg Oral Med Oral Pathol Oral Radiol
Endod 1997;84:16-21.

3. Sathasivam HP, Davies GR, Boyd NM. Predictive factors for osteoradionecrosis of the jaws: a retrospective study. Head Neck 2018;40:46-54.

4. Ramli R, Ngeow WC, Rahman RA, Chai WL. Managing complications of radiation therapy in head and neck cancer patients: part IV. Management of osteoradionecrosis. Singapore Dent J 2006;28:115 .

5. Regaud C. [Sur la necrose des os attente par un processus cancereux et traits par les radiations]. Compt Rend Soc Biol 1922; 87:629. French.

6. Marx RE. Osteoradionecrosis: a new concept of its pathophysiology. J Oral Maxillofac Surg 1983;41:283-8.

7. Watson WL, Scarborough JE. Osteoradionecrosis in intraoral cancer. Am J Roentgenol 1938;40:524-34.

8. Epstein JB, Rea G, Wong FL, Spinelli J, Stevenson-Moore P. Osteonecrosis: study of the relationship of dental extractions in patients receiving radiotherapy. Head Neck Surg 1987;10:48-54.

9. Thorn JJ, Hansen HS, Specht L, Bastholt L. Osteoradionecrosis of the jaws: clinical characteristics and relation to the field of irradiation. J Oral Maxillofac Surg 2000;58:1088-93; discussion 1093-5.

10. Jereczek-Fossa BA, Garibaldi C, Catalano G, d'Onofrio A, De Pas $\mathrm{T}$, Bocci $\mathrm{C}$, et al. Analysis of mandibular dose distribution in radiotherapy for oropharyngeal cancer: dosimetric and clinical results in 18 patients. Radiother Oncol 2003;66:49-56.

11. Maxymiw WG, Wood RE, Liu FF. Postradiation dental extractions without hyperbaric oxygen. Oral Surg Oral Med Oral Pathol 1991;72:270-4.

12. Katsura K, Sasai K, Sato K, Saito M, Hoshina H, Hayashi T. Relationship between oral health status and development of osteoradionecrosis of the mandible: a retrospective longitudinal study. Oral Surg Oral Med Oral Pathol Oral Radiol Endod 2008;105:731-8.

13. Piret P, Deneufbourg JM. [Mandibular osteoradionecrosis: sword of Damocles of radiotherapy for head and neck cancers?] Rev Med Liege 2002;57:393-9. French.

14. Jereczek-Fossa BA, Orecchia R. Radiotherapy-induced mandibular bone complications. Cancer Treat Rev 2002;28:65-74.

15. Epstein JB, Hatcher DC, Graham M. Bone scintigraphy of fibroosseous lesions of the jaw. Oral Surg Oral Med Oral Pathol 1981;51:346-50.

16. Mainous EG, Hart GB. Osteoradionecrosis of the mandible. Treatment with hyperbaric oxygen. Arch Otolaryngol 1975;101:173-7.

17. Scannapieco FA, Yu J, Raghavendran K, Vacanti A, Owens SI, Wood $\mathrm{K}$, et al. A randomized trial of chlorhexidine gluconate on oral bacterial pathogens in mechanically ventilated patients. Crit Care 2009;13:R117.

18. Støre G, Boysen M. Mandibular osteoradionecrosis: clinical behaviour and diagnostic aspects. Clin Otolaryngol Allied Sci 2000;25:378-84.

19. Gevorgyan A, Wong K, Poon I, Blanas N, Enepekides DJ, Higgins KM. Osteoradionecrosis of the mandible: a case series at a single institution. J Otolaryngol Head Neck Surg 2013;42:46.

20. Kuhnt T, Stang A, Wienke A, Vordermark D, Schweyen R, Hey J. Potential risk factors for jaw osteoradionecrosis after radiotherapy for head and neck cancer. Radiat Oncol 2016;11:101.

21. Kuo TJ, Leung CM, Chang HS, Wu CN, Chen WL, Chen GJ, et al. Jaw osteoradionecrosis and dental extraction after head and neck radiotherapy: a nationwide population-based retrospective study in Taiwan. Oral Oncol 2016;56:71-7.

22. Chronopoulos A, Zarra T, Tröltzsch M, Mahaini S, Ehrenfeld M, Otto S. Osteoradionecrosis of the mandible: a ten year single-center retrospective study. J Craniomaxillofac Surg 2015;43:837-46.

23. Koga DH, Salvajoli JV, Kowalski LP, Nishimoto IN, Alves FA. Dental extractions related to head and neck radiotherapy: ten-year experience of a single institution. Oral Surg Oral Med Oral Pathol Oral Radiol Endod 2008;105:e1-6.

24. Moon DH, Moon SH, Wang K, Weissler MC, Hackman TG, Zana- 
tion AM, et al. Incidence of, and risk factors for, mandibular osteoradionecrosis in patients with oral cavity and oropharynx cancers. Oral Oncol 2017;72:98-103.

25. Ben-David MA, Diamante M, Radawski JD, Vineberg KA, Stroup C, Murdoch-Kinch CA, et al. Lack of osteoradionecrosis of the mandible after intensity-modulated radiotherapy for head and neck cancer: likely contributions of both dental care and improved dose distributions. Int J Radiat Oncol Biol Phys 2007;68:396-402.

26. Nooh N. Dental implant survival in irradiated oral cancer patients: a systematic review of the literature. Int J Oral Maxillofac Implants 2013;28:1233-42.

27. Tsai CJ, Hofstede TM, Sturgis EM, Garden AS, Lindberg ME, Wei $\mathrm{Q}$, et al. Osteoradionecrosis and radiation dose to the mandible in patients with oropharyngeal cancer. Int J Radiat Oncol Biol Phys 2013;85:415-20.

28. Wahl MJ. Osteoradionecrosis prevention myths. Int J Radiat Oncol Biol Phys 2006;64:661-9.

29. Lyons A, Ghazali N. Osteoradionecrosis of the jaws: current understanding of its pathophysiology and treatment. Br J Oral Maxillofac Surg 2008; 46:653-60.

30. Walter C, Grötz KA, Kunkel M, Al-Nawas B. Prevalence of bisphosphonate associated osteonecrosis of the jaw within the field of osteonecrosis. Support Care Cancer 2007;15:197-202.

31. Jawad H, Hodson NA, Nixon PJ. A review of dental treatment of head and neck cancer patients, before, during and after radiotherapy: part 1. Br Dent J 2015;218:65-8.

32. Kluth EV, Jain PR, Stuchell RN, Frich JC Jr. A study of factors contributing to the development of osteoradionecrosis of the jaws. J Prosthet Dent 1988;59:194-201.

33. Owosho AA, Tsai CJ, Lee RS, Freymiller H, Kadempour A, Varthis $\mathrm{S}$, et al. The prevalence and risk factors associated with osteoradionecrosis of the jaw in oral and oropharyngeal cancer patients treated with intensity-modulated radiation therapy (IMRT): the Memorial Sloan Kettering Cancer Center experience. Oral Oncol 2017;64:44-51.

34. Curi MM, Dib LL. Osteoradionecrosis of the jaws: a retrospective study of the background factors and treatment in 104 cases. J Oral Maxillofac Surg 1997;55:540-4; discussion 545-6.

35. Malska-Waniewska I. Microdensitometric analysis of the influence of $\mathrm{x}$-irradiation on mature bone in humans. Acta Med Pol 1971;12:357-80.

36. Marx RE, Johnson RP, Kline SN. Prevention of osteoradionecrosis: a randomized prospective clinical trial of hyperbaric oxygen versus penicillin. J Am Dent Assoc 1985;111:49-54. 